\title{
Identification du bacille de la maladie de Whipple?
}

Dans le premier cas publié - en 1907 - de la maladie qui porte son nom, G. H. Whipple [1] avait observé de très nombreux micro-organismes, en forme de bâtonnets, dans un ganglion mésentérique, et suggéré qu'ils pourraient être l'agent causal de cette affection (initialement dénommée " lipodystrophie intestinale ", elle est en fait systémique, intéressant surtout le tractus gastro-intestinal, le mésentère, le cœur et le système nerveux central, frappe plus souvent l'homme d'âge mûr, et se traduit cliniquement par arthralgies, diarrhée, douleurs abdominales, amaigrissement, fièvre, adénopathies et pigmentation cutanée). Ce n'est qu'en 1961 que ces bâtonnets furent identifiés, en microscopie électronique, à des bacilles de siège extracellulaire et intra-macrophagique ; dans ces dernières cellules, le matériel PAS + - si caractéristique des lésions, en particulier intestinales, de la maladie - correspond à des bactéries intactes ou en dégradation. Malgré la sensibilité clinique, rapide et spectaculaire, de la maladie à l'antibiothérapie, et alors que disparaissent les bactéries extra-cellulaires, l'infiltration bactérienne macrophagique persiste, quant à elle, très longtemps voire définitivement sous traitement. De multiples essais de mise en culture du "bacille de Whipple ", in vitro ou dans des tissus animaux, se sont avérés infructueux ; d'autres, jugés positifs, ont isolé des espèces fort différentes, dont Streptococcus dysgalactiae et Corynebacterium bovis ont été parmi les dermières en date $[2,3]$.

Le développement d'une nouvelle technique d'identification bactérienne fondée sur l'amplification de l'ARN ribosomique (ARNr) 16S et la comparaison de sa séquence nucléotidique à des séquences répertoriées, ce qui est une puissante méthode d'analyse $m / s n^{\circ} 8$, vol. 8, octobre 92 phylogénétique de toutes les formes de vie - avait déjà permis à Relman $e t$ al. [4] d'identifier l'agent vraisemblable de l'angiomatose bacillaire, et à Wilson et al. [5] de suggérer, mais à partir d'un seul cas, que le bacille de Whipple serait "phylogénétiquement " proche d'un nocardioforme, Rhodococcus equi. Relmann et al. [6] ont, à nouveau, récemment appliqué cette technique aux tissus de cinq sujets atteints de maladie de Whipple, vivant dans des régions différentes des États-Unis et n'ayant eu aucun contact entre eux. A partir de la muqueuse duodénale du premier malade, ils ont amplifié une séquence bactérienne d'ARNr $16 \mathrm{~S}$ de 1321 bases. Ils ont ensuite détecté cette séquence dans la muqueuse duodénale et les ganglions (rétropéritonéaux, mésentériques ou supra-claviculaires) des patients $2,3,4$ et 5 , mais pas dans divers tissus - gastro-duodénal, ganglionnaire périphérique, cutané, rénal, splénique ou amygdalien - de dix sujets contrôles indemnes de maladie de Whipple (deux de ces contrôles avaient une angiomatose bacillaire associée dans un cas à une infection à Mycobacterium avium - et un avait une maladie des griffes de chat). Selon cette analyse phylogénétique, la bactérie de la maladie de Whipple est proche du groupe des actinomycètes dont la plupart sont des microorganismes telluriques, aérobies et Gram + , jugés saprophytes - , mais ne correspond à aucun genre connu : elle a été dénommée Tropheryma whippelii.

G. H. Whipple avait souligné la similarité de la maladie avec ce que l'on connaissait, alors, des mycobactérioses gastro-intestinales. Bien que proche de ce groupe d'organismes, T. whippelii n'est pas, directement, un nocardioforme ; néanmoins, il est intéressant de se souvenir que des nocardioformes du type Mycobacterium paratuberculosis, Mycobacterium avium complex, et Rhodococcus equi ont tous été notés, chez l'animal comme chez des sujets infectés par le virus $\mathrm{HIV}$, en association à des lésions ressemblant de très près à celles de la maladie de Whipple; la nature inhabituelle de leur paroi pourrait être un trait pathogénique commun à ces micro-organismes chez certains hôtes immunodéficients.

Il reste à savoir si $T$. whippelii est une espèce rare de la flore microbienne normale de l'homme et s'il peut s'associer, chez lui, à d'autres affections ; est à souligner la fréquence, au cours de la maladie de Whipple, de lésions rappelant celles de la sarcoïdose. Finalement, ce travail ouvre réellement une voie dans le labyrinthe pathogénique de la maladie, et pourrait fournir un outil de diagnostic précoce et spécifique.

C. M.

1. Whipple GH. A hitherto undescribed disease characterized anatomically by deposits of fat and fatty acids in the intestinal and mesenteric lymphatic tissues. Bull Johns Hopkins Hosp 1907 ; 18 : 382-91.

2. Clancy TR, Tomkins WAF, Muckle TJ, Richardson $\mathrm{H}$, Rawls WE. Isolation and characterization of an etiological agent in Whipple's disease. BMJ 1975: 3: 568-70.

3. Gupta S, Pinching AJ, Onwubalili J, Vince A, Evans DJ, Hodgson HJF. Whipple's disease with unusual clinical, bacteriologic and immunologic findings. Gastroenterology $1986 ; 90$ : 1286-9. 4. Relman DA, Loutit JS, Schmidt TM, Falkow S, Tompkins LS. The agent of bacillary angiomatosis - an approach to the identification of uncultured pathogens. $N$ Engl J Med $1990 ; 323$ : 1573-80.

5. Wilson $\mathrm{KH}$, Blitchington $\mathrm{R}$, Frothingham $\mathrm{R}$, Wilson JAP. Phylogeny of the Whipple's-diseaseassociated bacterium. Lancet 1991 ; 338 : 474-5. 6. Relman DA, Schmidt TM, MacDermott RP, Falkow S. Identification of the uncultured bacillus of Whipple's disease. N Engl J Med 1992 ; 327 : 293-301.



